\title{
Approximative analytical solutions of the Dirac equation in Schwarzschild spacetime
}

\author{
Ion I. Cotăescu * \\ West University of Timişoara, \\ V. Pârvan Ave. 4, RO-300223 Timişoara, Romania
}

September 24, 2018

\begin{abstract}
Approximative analytic solutions of the Dirac equation in the geometry of Schwarzschild black holes are derived obtaining information about the discrete energy levels and the asymptotic behavior of the energy eigenspinors.

Pacs: 04.62.+v
\end{abstract}

The problem of the Dirac perturbations in the gravitational field of a Schwarzschild black hole is less studied since the free Dirac equation in this geometry is not analytically solvable at least in the systems of coordinates and tetrad gauge fixings used until now. However, despite of this difficulty, interesting results were obtained using suitable analytical and numerical methods [1]-[3].

In this letter we would like to consider this problem in a new conjecture in which the local frames of the central static charts are defined by the tetrad fields we have proposed some time ago $[4,5]$. This tetrad gauge shows off the central symmetry as a global one such that the separation of the spherical variables can be done as in special relativity [6]. After the separation of the angular variables we remain with a pair of simple radial equations depending

*E-mail: cota@physics.uvt.ro 
only on the metric components. In this approach we have found the energy eigenspinors of a massive Dirac field on the de-Sitter [7] and anti-de Sitter spacetimes $[4,8]$. Unfortunately, this can not be achieved in the case of the Schwarzschild background.

For this reason, our purpose is to find reasonable minor approximations of the radial equations in which these could be analytically solvable. In this way we obtain interesting information concerning the discrete energy levels and the asymptotic behavior of the energy eigenspinors. The results are presented in natural units with $\hbar=c=1$.

In general, a manifold with central symmetry has a central static chart with spherical coordinates $(t, r, \theta, \phi)$, covering the space domain $D=D_{r} \times S^{2}$, i.e. $r \in D_{r}$ while $\theta$ and $\phi$ cover the sphere $S^{2}$. For defining the local frames we use the tetrad fields defined in Refs. $[4,5]$ whose components depend on three arbitrary functions of $r$, denoted by $u, v$ and $w$, which give the line element as

$$
d s^{2}=w^{2}\left[d t^{2}-\frac{d r^{2}}{u^{2}}-\frac{r^{2}}{v^{2}}\left(d \theta^{2}+\sin ^{2} \theta d \phi^{2}\right)\right] .
$$

We have shown [4] that in these circumstances the separation of the spherical variables of the free Dirac equation can be done as in the case of the central problems in Minkowski flat spacetime. Consequently, the Dirac field of mass $m$ can be written as a linear combination of particular solutions of given energy. Those of positive frequency and energy $E$,

$$
\begin{aligned}
U_{E, j, m_{j}, \kappa_{j}}(x) & =U_{E, j, m_{j}, \kappa_{j}}(t, r, \theta, \phi) \\
& =\frac{v(r)}{r w(r)^{3 / 2}}\left[f^{+}(r) \Phi_{m_{j}, \kappa_{j}}^{+}(\theta, \phi)+f^{-}(r) \Phi_{m_{j}, \kappa_{j}}^{-}(\theta, \phi)\right] e^{-i E t},
\end{aligned}
$$

are particle-like energy eigenspinors expressed in terms of radial wave functions $f^{ \pm}$and usual four-component angular spinors $\Phi_{m_{j}, \kappa_{j}}^{ \pm}$. These spinors are orthogonal to each other being completely determined by the angular quantum numbers, $j$ and $m_{j}$, and the value of $\kappa_{j}= \pm(j+1 / 2)[6,9]$. Moreover, they are normalized to unity with respect to their own angular scalar product. We note that the antiparticle-like energy eigenspinors can be obtained directly using the charge conjugation as in the flat case [8].

Thus the problem of the angular motion is completely solved. We remain with the radial wave functions $f^{ \pm}$which satisfy two radial equations that can be written in compact form as the eigenvalue problem $H \mathcal{F}=E \mathcal{F}$ of the 
radial Hamiltonian [4]

$$
H=\left|\begin{array}{cc}
m w & -u \frac{d}{d r}+\kappa_{j} \frac{v}{r} \\
u \frac{d}{d r}+\kappa_{j} \frac{v}{r} & -m w
\end{array}\right|,
$$

in the space of two-component vectors, $\mathcal{F}=\left|f^{+}, f^{-}\right|^{T}$, equipped with the radial scalar product [4]

$$
\left(\mathcal{F}_{1}, \mathcal{F}_{2}\right)=\int_{D_{r}} \frac{d r}{u} \mathcal{F}_{1}^{\dagger} \mathcal{F}_{2}
$$

This selects the 'good' radial wave functions, i.e. square integrable functions or tempered distributions, which enter in the structure of the particle-like energy eigenspinors (2).

Let us consider now a Dirac particle of mass $m$ freely moving in the gravitational field of a black hole of mass $M$ with the Schwarzschild line element

$$
d s^{2}=\left(1-\frac{r_{0}}{r}\right) d t^{2}-\frac{d r^{2}}{1-\frac{r_{0}}{r}}-r^{2}\left(d \theta^{2}+\sin ^{2} \theta d \phi^{2}\right)
$$

defined on the radial domain $D_{r}=\left(r_{0}, \infty\right)$ where $r_{0}=2 M G$. Hereby we identify the functions

$$
u(r)=1-\frac{r_{0}}{r}, \quad v(r)=w(r)=\sqrt{1-\frac{r_{0}}{r}},
$$

that give the radial Hamiltonian (3). The resulting radial problem can not be analytically solved as it stays. Therefore, if we desire to avoid the numerical methods, we are forced to look for suitable approximations of the radial equations.

In order to find an efficient method of approximation it is convenient to introduce the new dimensionless variable

$$
x=\sqrt{\frac{r}{r_{0}}-1} \in(0, \infty)
$$

and the notations

$$
\mu=r_{0} m, \quad \epsilon=r_{0} E
$$


which allow us to rewrite the radial problem as

$$
\left|\begin{array}{cc}
\mu \sqrt{1+x^{2}}-\epsilon\left(x+\frac{1}{x}\right) & -\frac{1}{2} \frac{d}{d x}+\frac{\kappa_{j}}{\sqrt{1+x^{2}}} \\
\frac{1}{2} \frac{d}{d x}+\frac{\kappa_{j}}{\sqrt{1+x^{2}}} & -\mu \sqrt{1+x^{2}}-\epsilon\left(x+\frac{1}{x}\right)
\end{array}\right|\left|\begin{array}{l}
f^{+}(x) \\
f^{-}(x)
\end{array}\right|=0 .
$$

Moreover, from Eq.(4) we find that the radial scalar product in the new variable takes the form

$$
\left(\mathcal{F}_{1}, \mathcal{F}_{2}\right)=2 r_{0} \int_{0}^{\infty} d x\left(x+\frac{1}{x}\right) \mathcal{F}_{1}^{\dagger} \mathcal{F}_{2}
$$

Near singularity, for small values of $x \sim 0$, the exact solutions can be approximated by $\mathcal{F}_{s}=\left|f_{s}^{+}, f_{s}^{-}\right|^{T}$ which satisfy the approximative radial equations,

$$
\left|\begin{array}{cc}
\mu-\frac{\epsilon}{x} & -\frac{1}{2} \frac{d}{d x}+\kappa_{j} \\
\frac{1}{2} \frac{d}{d x}+\kappa_{j} & -\mu-\frac{\epsilon}{x}
\end{array}\right|\left|\begin{array}{c}
f_{s}^{+}(x) \\
f_{s}^{-}(x)
\end{array}\right|=0
$$

where we neglected the terms of the order $O(x)$. Performing the transformation $\mathcal{F}_{s} \rightarrow \hat{\mathcal{F}}_{s}=U \mathcal{F}_{s}=\left|\hat{f}_{s}^{+}, \hat{f}_{s}^{-}\right|^{T}$, with the help of the unitary matrix

$$
U=\frac{1}{\sqrt{2}}\left|\begin{array}{ll}
1 & i \\
i & 1
\end{array}\right|
$$

we obtain the simpler system of equations

$$
\left(\frac{1}{2} \frac{d}{d x} \pm \frac{i \epsilon}{x}\right) \hat{f}_{s}^{ \pm}(x)=\left(\mu \pm i \kappa_{j}\right) \hat{f}_{s}^{\mp}(x) .
$$

This can be analytically solved in terms of Bessel functions $J_{l}$ and $Y_{l}$ as [10]

$$
\begin{aligned}
& \hat{f}_{s}^{+}(x)=\sqrt{\kappa_{j}-i \mu}\left(c_{1} \sqrt{x} J_{l_{+}}\left(2 i \lambda_{j} x\right)+c_{2} \sqrt{x} Y_{l_{+}}\left(2 i \lambda_{j} x\right)\right), \\
& \hat{f}_{s}^{-}(x)=\sqrt{\kappa_{j}+i \mu}\left(c_{1} \sqrt{x} J_{l_{-}}\left(2 i \lambda_{j} x\right)+c_{2} \sqrt{x} Y_{l_{-}}\left(2 i \lambda_{j} x\right)\right),
\end{aligned}
$$

where $c_{1}$ and $c_{2}$ are arbitrary constants and

$$
l_{ \pm}=2 i \epsilon \pm \frac{1}{2}, \quad \lambda_{j}=\sqrt{\kappa_{j}^{2}+\mu^{2}} .
$$


We observe that near singularity these wave functions are regular but their phases are not determined since $\hat{f}_{s}^{ \pm} \propto x^{\mp 2 i \epsilon}+O(x)$ for $x \rightarrow 0$.

The asymptotic behavior, for very large values of $x$, can be studied approximating $\sqrt{1+x^{2}} \sim x$ and obtaining thus an asymptotic radial problem for $\mathcal{F}_{a}=\left|f_{a}^{+}, f_{a}^{-}\right|^{T}$. The asymptotic radial equations can be put in the form

$$
\left|\begin{array}{cc}
\frac{1}{2} \frac{d}{d x}+\frac{\kappa_{j}}{x} & -\mu\left(x+\frac{\delta}{x}\right)-\epsilon\left(x+\frac{1}{x}\right) \\
-\mu\left(x+\frac{\delta}{x}\right)+\epsilon\left(x+\frac{1}{x}\right) & \frac{1}{2} \frac{d}{d x}-\frac{\kappa_{j}}{x}
\end{array}\right|\left|\begin{array}{l}
f_{a}^{+}(x) \\
f_{a}^{-}(x)
\end{array}\right|=0,
$$

where we introduced the new parameter $\delta \in[0,1)$ which could play the role of a fit parameter. Of course, if we rigorously consider the approximation of the order $O(1 / x)$ then we must take $\delta=\frac{1}{2}$.

Since Eqs. (17) can be solved for any value of $\epsilon$, we have to look separately for solutions corresponding either to a discrete energy spectrum when $\epsilon<\mu$ or to a continuous one in the domain $[\mu, \infty)$. For finding these solutions, we diagonalize the term proportional to $x$ of the operator (17) using the transformation matrix

$$
T=\frac{1}{2 \nu}\left|\begin{array}{cc}
\sqrt{\mu-\epsilon} & \sqrt{\mu+\epsilon} \\
-\sqrt{\mu-\epsilon} & \sqrt{\mu+\epsilon}
\end{array}\right|
$$

where we denote $\nu=\sqrt{\mu^{2}-\epsilon^{2}}$. After the transformation $\mathcal{F}_{a} \rightarrow \hat{\mathcal{F}}_{a}=T \mathcal{F}_{a}=$ $\left|\hat{f}_{a}^{+}, \hat{f}_{a}^{-}\right|^{T}$ we obtain the new equations

$$
\left[\frac{1}{2} x \frac{d}{d x} \pm\left(\frac{\epsilon^{2}-\delta \mu^{2}}{\nu}-\nu x^{2}\right)\right] \hat{f}_{a}^{ \pm}=\left(\kappa \mp \frac{\epsilon \mu(\delta-1)}{\nu}\right) \hat{f}_{a}^{\mp},
$$

that can be analytically solved.

The discrete quantum modes with discrete energy levels $\epsilon<\mu$ are governed by radial wave functions which must be square integrable. After a little calculation we find that Eqs. (19) allow the particular solutions

$$
\begin{aligned}
& \hat{f}_{a}^{+}(x)=N^{+} x^{2 s_{j}} e^{-\nu x^{2}}{ }_{1} F_{1}\left(s_{j}+q+1,2 s_{j}+1,2 \nu x^{2}\right), \\
& \hat{f}_{a}^{-}(x)=N^{-} x^{2 s_{j}} e^{-\nu x^{2}}{ }_{1} F_{1}\left(s_{j}+q, 2 s_{j}+1,2 \nu x^{2}\right),
\end{aligned}
$$

where the confluent hypergeometric functions ${ }_{1} F_{1}[10]$ depend on the following real parameters

$$
s_{j}=\sqrt{\kappa_{j}^{2}+\nu^{2}+\mu^{2}\left(\delta^{2}-1\right)}, \quad q=\nu+\frac{\mu^{2}(\delta-1)}{\nu} .
$$


These solutions satisfy Eqs. (19) only if the normalization constants $N^{ \pm}$ obey

$$
\frac{N^{-}}{N^{+}}=\frac{\nu\left(s_{j}-q\right)}{\kappa_{j} \nu-\mu \epsilon(\delta-1)} .
$$

We obtain thus the transformed radial wave functions (20) and (21) that have a good asymptotic behavior and are regular in $x=0$.

These solutions may be square integrable with respect to the scalar product (10) only when we impose the quantization condition $s_{j}+q=-n, n=$ $1,2,3, \ldots$. This leads to the equation

$$
\sqrt{\kappa_{j}^{2}+\nu^{2}+\mu^{2}\left(\delta^{2}-1\right)}+\nu+\frac{\mu^{2}(\delta-1)}{\nu}=-n,
$$

which is of the third order in $\nu$ and can be solved at anytime under Mathematica or Maple, obtaining thus the asymptotic energy spectrum. However, the analytic formula of these energy levels is too complicated to be written here. For this reason, we restrict ourselves to give some reasonable estimations. First we observe that the energies levels, $E_{n, j}$, must satisfy the conditions

$$
\frac{\kappa_{j}^{2}}{\kappa_{j}^{2}+\mu^{2}(1-\delta)^{2}} \leq \frac{E_{n, j}{ }^{2}}{m^{2}}<1 \leq \delta^{2}+\frac{\kappa_{j}^{2}}{\mu^{2}} .
$$

The last inequality prevents the Dirac particles to get near to the singularity where the black hole could capture them. For $\mu<1$ and small values of $\nu$ we have to use the approximative formula

$$
E_{n, j} \simeq m\left[1-\frac{\mu^{2}(1-\delta)^{2}}{\left(n+\sqrt{\kappa_{j}^{2}+\mu^{2}\left(\delta^{2}-1\right)}\right)^{2}}\right]^{\frac{1}{2}} .
$$

Moreover, in the case of $\mu \ll 1$ and $\delta=\frac{1}{2}$ we recover the Newtonian result

$$
E_{n, j} \sim m\left(1-\frac{1}{8} \frac{\mu^{2}}{\left(n+j+\frac{1}{2}\right)^{2}}\right)=m-\frac{1-e^{2}}{2} \frac{G^{2} M^{2} m^{3}}{L^{2}} .
$$

for a particle of mass $m=\mu / r_{0}$ and angular momentum $L=j \pm \frac{1}{2}$ moving on an ellipsoidal trajectory of eccentricity

$$
e=\left(1-\frac{\left(j \pm \frac{1}{2}\right)^{2}}{\left(n+j+\frac{1}{2}\right)^{2}}\right)^{\frac{1}{2}} .
$$


These asymptotical results, including the exact solutions of Eq. (24), must be considered prudently trying to verify and improve them using numerical methods. We note that only the massive fermions can be retained in Schwarzschild gravitational fields on discrete levels while the massless fermions can not do this.

The scattering modes corresponding to the continuous energy spectrum $\epsilon \in[\mu, \infty)$ are described by particular solutions that behave as tempered distributions. In this case we introduce the real parameter $\hat{\nu}=\sqrt{\epsilon^{2}-\mu^{2}}$ instead of $\nu$. Following the same procedure like in the previous case we look for the solutions of the transformed equations (19) where we replace $\nu=i \hat{\nu}$. Hereby we obtain the transformed radial wave functions in terms of Whittaker functions $M_{p, s}$ and $W_{p, s}$ as [10]

$$
\begin{aligned}
& \hat{f}_{a}^{+}(x)=C_{1}^{+} \frac{1}{x} M_{p_{+}, s_{j}}\left(2 i \hat{\nu} x^{2}\right)+C_{2}^{+} \frac{1}{x} W_{p_{+}, s_{j}}\left(2 i \hat{\nu} x^{2}\right) \\
& \hat{f}_{a}^{-}(x)=C_{1}^{-} \frac{1}{x} M_{p_{-}, s_{j}}\left(2 i \hat{\nu} x^{2}\right)+C_{2}^{-} \frac{1}{x} W_{p_{-}, s_{j}}\left(2 i \hat{\nu} x^{2}\right),
\end{aligned}
$$

where we denote $p_{ \pm}=\mp \frac{1}{2}-q$ while the constants satisfy

$$
\frac{C_{1}^{-}}{C_{1}^{+}}=\frac{\hat{\nu}\left(s_{j}-q\right)}{\kappa_{j} \hat{\nu}+i \epsilon \mu(\delta-1)}, \quad \frac{C_{2}^{-}}{C_{2}^{+}}=-\frac{\hat{\nu}}{\kappa_{j} \hat{\nu}+i \epsilon \mu(\delta-1)} .
$$

These solutions are useful for analyzing the scattering of the Dirac particles on black holes.

Hence we have all the elements we need to write down the approximative form of the energy eigenspinors in all of the above discussed cases. Starting with the approximative form of the transformed radial wave functions $\hat{f}^{ \pm}$we have to calculate the functions $f^{ \pm}$which should give the particle-like energy eigenspinors (2). The antiparticle-like energy eigenspinors can be calculated using the charge conjugation as [8]

$$
V_{E, j, m_{j}, \kappa_{j}}=\left(U_{E, j, m_{j}, \kappa_{j}}\right)^{c} \equiv C\left(\bar{U}_{E, j, m_{j}, \kappa_{j}}\right)^{T}, \quad C=i \gamma^{2} \gamma^{0}
$$

We hope that the approximative solutions presented here should help one to improve the analytical or numerical methods for studying the Dirac field in the Schwarzschild background. 


\section{References}

[1] J. Jing, Phys. Rev. D 70, 065004 (2004); Phys. Rev. D 71, 124006 (2005).

[2] K. H. C. Castello-Branco, R. A. Konoplya and A. Zhidenko, Phys. Rev. D 71, 047502 (2005).

[3] S. Dolan,C. Doran and A. Lasenby, Phys. Rev. D 74, 064005 (2006).

[4] I. I. Cotăescu, Mod. Phys. Lett. A 13, 2923 (1998)

[5] I. I. Cotăescu, J. Phys. A: Math. Gen. 33, 1977 (2000).

[6] B. Thaller, The Dirac Equation, Springer Verlag, Berlin Heidelberg, 1992

[7] I. I. Cotăescu, Mod. Phys. Lett. A 13, 2991 (1998)

[8] I. I. Cotăescu, Phys. Rev. D 60, 124006 (1999).

[9] J. D. Bjorken and S. D. Drell, Relativistic Quantum Mechanics, McGraw-Hill Book Co., NY, 1964

[10] M. Abramowitz and I. A. Stegun, Handbook of Mathematical Functions Dover, 1964 